Schwerpunkt

Kardiologe 2020 $14: 113-123$

https://doi.org/10.1007/s12181-020-00388-y

Online publiziert: 13. März 2020

(c) Deutsche Gesellschaft für Kardiologie Herz- und Kreislaufforschung e.V. Published by Springer Medizin Verlag $\mathrm{GmbH}$, ein Teil von Springer Nature - all rights reserved 2020

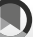

Dominic Millenaar ${ }^{1} \cdot$ Tobias Fehlmann $^{2} \cdot$ Christian Ukena $^{1} \cdot$ Sean Scholz' . Andreas Keller ${ }^{2}$. Felix Mahfoud' $\cdot$ Michael Böhm ${ }^{1}$

' Klinik für Innere Medizin III, Kardiologie, Angiologie und Internistische Intensivmedizin, Universitätsklinikum des Saarlandes und Medizinische Fakultät, Universität des Saarlandes, Homburg/Saar, Deutschland

${ }^{2}$ Zentrum für Bioinformatik Saar, Abteilung für klinische Bioinformatik, Universität des Saarlandes, Saarbrücken, Deutschland

\title{
Wissenschaftslandschaft Deutschland
}

de die Qualität wissenschaftlicher Publikationen durch den Hirsch(H)-Index als Maß der wissenschaftlichen Qualität herangezogen. Der H-Index beschreibt die Anzahl Publikationen eines Autors, die alle mindestens ebenso häufig zitiert wurden wie die Gesamtanzahl seiner Publikationen [3]. Der H-Index wurde ursprünglich zur Beurteilung der Forschungsleistung einzelner Autoren entwickelt, kann jedoch auch im Sinne ei- nes modifizierten $\mathrm{H}$-Indexes für ganze Forschungseinrichtungen, Bundesländer oder Länder angepasst werden, wie in der folgenden Analyse erfolgt. Im Weiteren wurde die Forschungsleistung sowohl nach unterschiedlichen Bundesländern als auch nach einzelnen Themenbereichen ausgewertet.

\section{Kardiologische Forschung in Deutschland}

In der kardiovaskulären Medizin sind ein Fortschritt und ein Zuwachs der Forschungsleistungen weltweit zu verzeichnen. Die steigende Prävalenz kardiovaskulärer Erkrankungen hat das wissenschaftliche Interesse aktiviert, um durch die Entwicklung neuer medikamentöser und interventioneller Therapieverfahren der steigenden Prävalenz kardiovaskulärer Erkrankungen Rechnung zu tragen. Ziel der vorliegenden Analyse ist, die Entwicklung der kardiovaskulären Forschung in Deutschland seit der Wiedervereinigung zu analysieren. Hierbei wur-

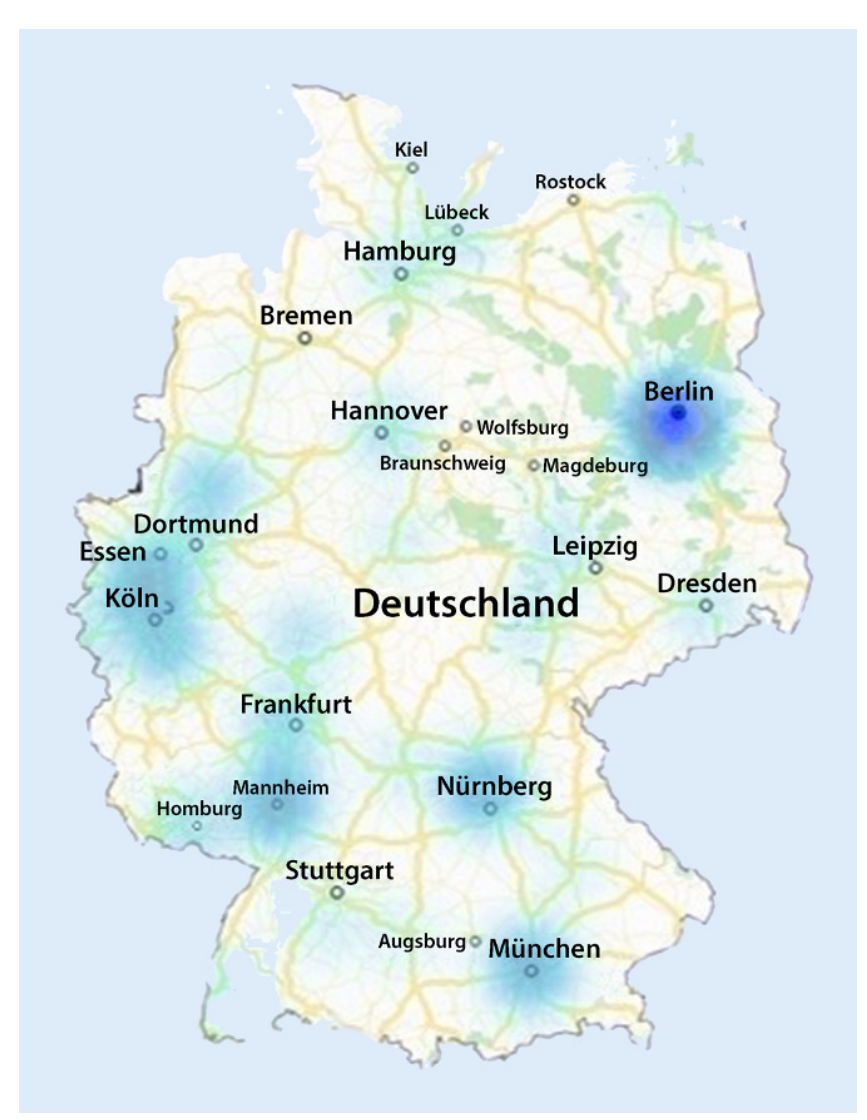

Abb. $1<$ Heatmap aller Institute in Deutschland. Die Farbintensität ist proportional zur Anzahl der kardiologischen Publikationen der entsprechenden Forschungseinrichtungen 

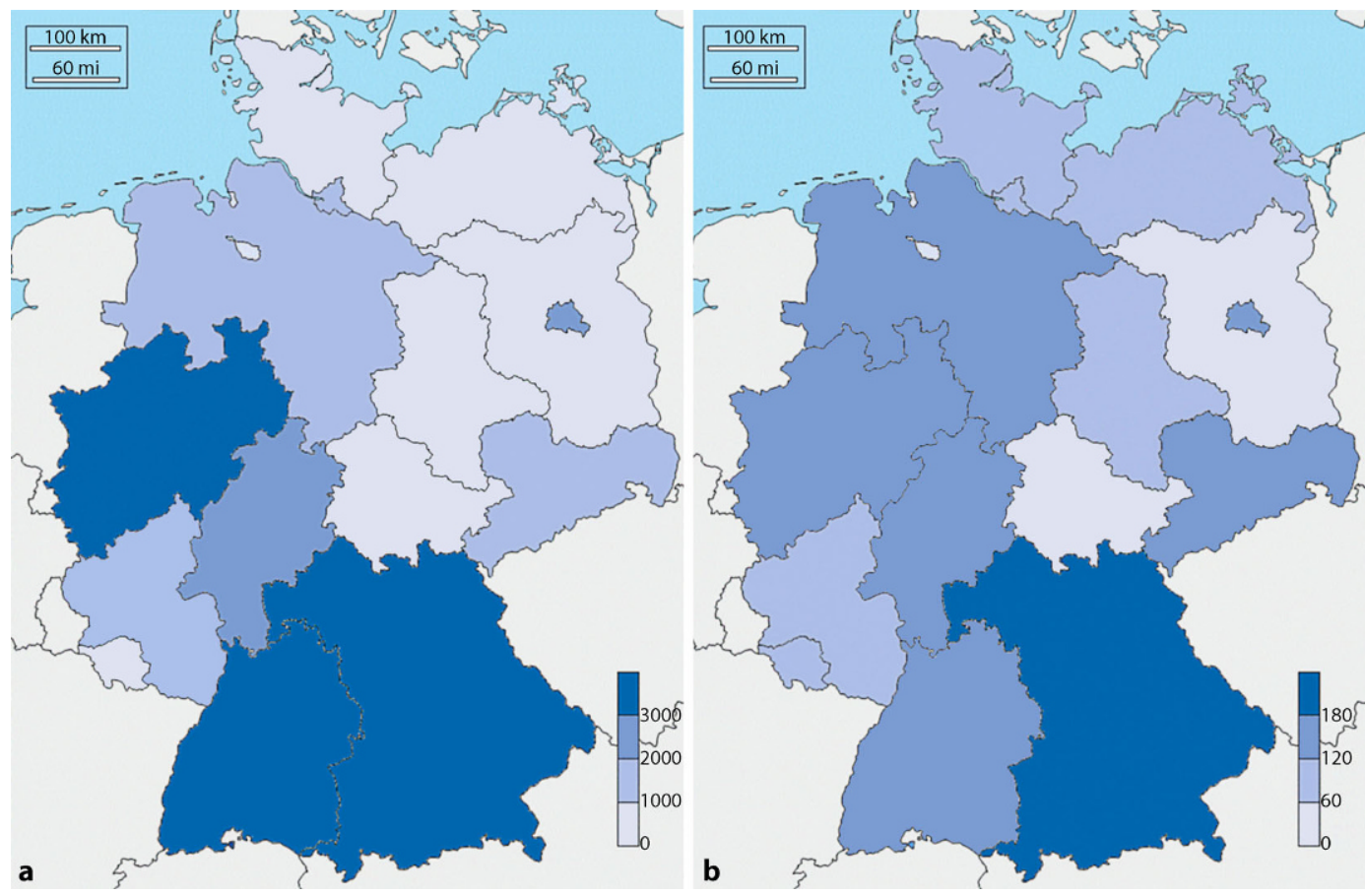

Abb. $2 \triangleleft$ Darstellung der Forschungsleistungen der einzelnen Bundesländer aller kardiologischen Publikationen bis 2018 in Bezug auf Gesamtanzahl der Publikationen (a) sowie des modifizierten Hirsch(H)-Indexes pro Bundesland (b). Farbkodierung entsprechend der jeweiligen Legende
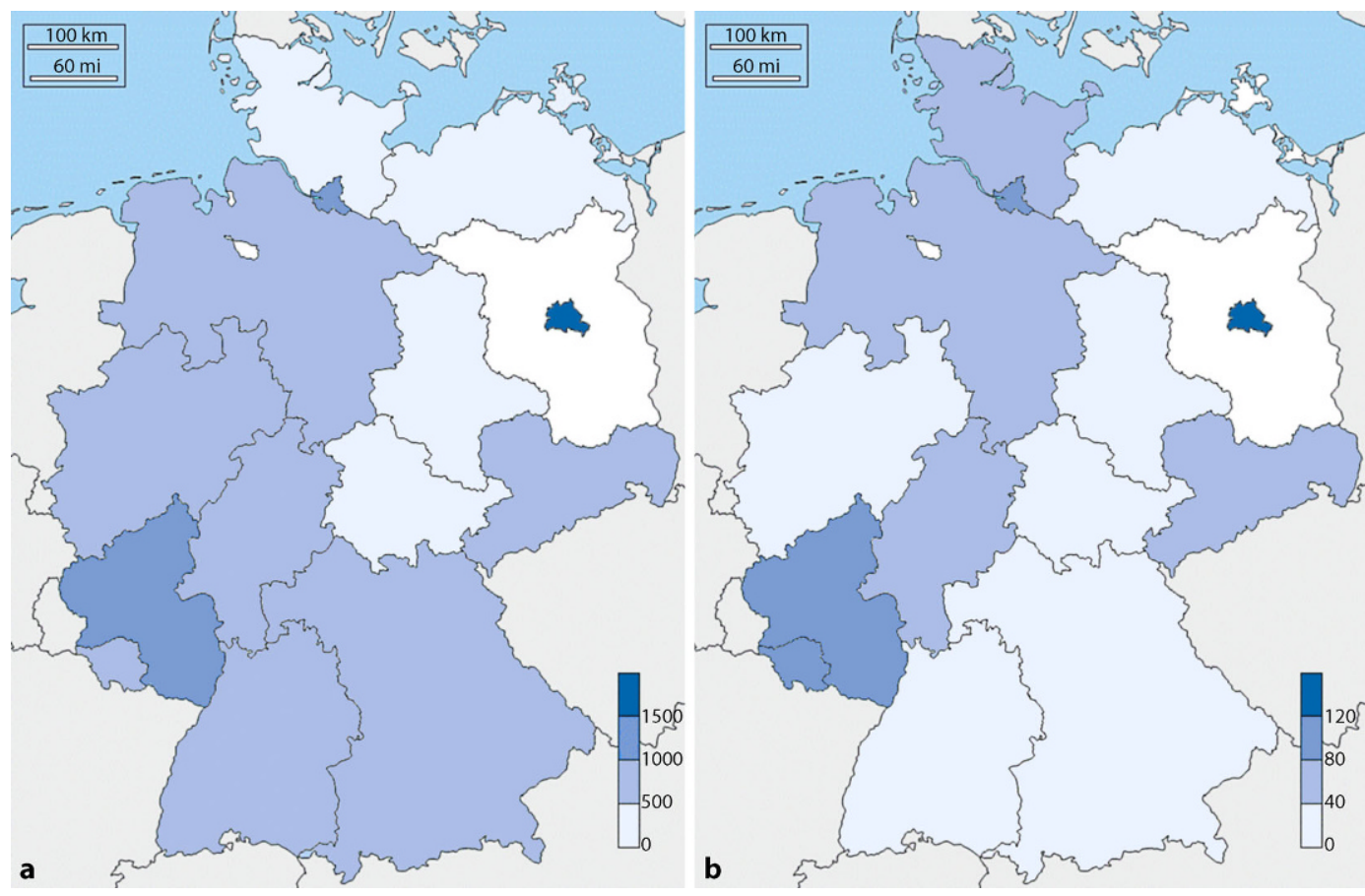

Abb. $3<$ Darstellung der Gesamtanzahl an kardiologischen Publikationen (a) sowie des modifizierten Hirsch(H)-Indexes (b) in Relation zur Anzahl der medizinischen Fakultät des jeweiligen Bundeslandes bis 2018. Farbkodierung entsprechend der jeweiligen Legende

\section{Publikationen}

Es wurden insgesamt alle Artikel der Kardiologie bis 2018 analysiert. Hieraus ergaben sich eine Gesamtzahl von kardiologischen Publikationen weltweit von 996.900 und eine Gesamtzahl von Publikationen aus deutschen wissenschaftlichen Instituten von 51.430. Dies entspricht einem Anteil von 5,2\% der gesamten kardiologischen Publikationsleistung weltweit. Mithilfe einer szientometrischen Analyse [4] wurden alle Publikationen geografisch den Instituten der federführenden Autoren zugeordnet und auf einer Deutschlandkarte in einer Heatmap in - Abb. 1 visualisiert. Die farbliche Kodierung gibt die Gesamtanzahl der Publikationen pro Institut über den gesamten Zeitraum bis 2018 an.

\section{Szientometrische Analyse}

Für diese szientometrische Analyse wurde zunächst ein Suchbegriff erstellt, der möglichst alle kardiologischen Veröffentlichungen abbildet und zugleich keine fachfremden Publikationen mit einbezieht. Hierbei wurden sowohl gängige Abkürzungen als auch verschiedene Sprachen berücksichtigt. Die Su- 
che erfolgte in der Online-Datenbank Web of Science Core Collection (WoS) mittels Schlagwortsuche in den Titeln der Artikel; berücksichtigt wurden nur Originalarbeiten aus deutschen Forschungseinrichtungen. Im Folgenden wird ein Ausschnitt des Suchterminus dargestellt (der gesamte Suchbegriff ist im Zusatzmaterial online aufgeführt): $\left[\mathrm{TI}=\left(\left(^{*}\right.\right.\right.$ cardy $^{\star} \mathrm{OR}^{*}{ }^{\star}$ cardi ${ }^{\star}$ OR heart OR coron ${ }^{\star}$ OR CVD OR CHF) OR (pacemaker AND (Permanent OR transvenous OR ${ }^{*}$ card $^{*}$ OR leadless) NOT (intracereb $^{\star}$ OR Parkinson OR neuronal OR nerv $\left.\left.^{\star}\right)\right)$ OR ((„art* hypertens ${ }^{\star *}$ OR hypertens ${ }^{\star}$ OR „blood pressure“ OR „isolated systolic hypertension“ OR „resistant hypertension“ OR ABPM) NOT intrac* NOT pulm* NOT portal NOT ocular NOT compartment $\left.{ }^{*}(\ldots)\right)$ ]. Zur weiteren Analyse wurden alle gefundenen Artikel szientometrisch analysiert mithilfe der Webanwendung „Science Performance Evaluation" (SciPE), die in Kooperation durch das Institut für Bioinformatik (Universität des Saarlandes, Saarbrücken) sowie der Klinik für Innere Medizin III (Kardiologie, Angiologie, Internistische Intensivmedizin, Universitätsklinikum des Saarlandes, Homburg/Saar) entwickelt wurde. Nach einer anfänglichen Datenverifikation durch SciPE erfolgen eine Analyse der Datensätze mit Geokodierung und somit eine geografische Zuordnung aller wissenschaftlichen Institute weltweit mit einer konsekutiven Visualisierung in einer Heatmap aller Institute (• Abb. 1). Weiterhin erfolgten Analysen der Autoren hinsichtlich Geschlechtes, Nationalität und Kooperationen zwischen allen Instituten und Ländern. Außerdem konnten alle Daten im zeitlichen Verlauf dargestellt werden, wie beispielsweise in

- Abb. 7 gezeigt.

\section{Publikationsqualität}

In - Abb. 2 ist eine Analyse der Forschungsleistung aller Bundesländer dargestellt. Alle Daten der - Abb. 2, 3, 4 und 5 basieren auf den Zahlen der $\bullet$ Tab. 1 und 2. In - Abb. 2a wird die Gesamtanzahl aller Publikationen pro Bundesland farbkodiert wiedergegeben. Hierbei wird deutlich, dass die Anzahl der Publika-

Kardiologe 2020 · 14:113-123 https://doi.org/10.1007/s12181-020-00388-y

(c) Deutsche Gesellschaft für Kardiologie - Herz- und Kreislaufforschung e.V. Published by Springer Medizin Verlag GmbH, ein Teil von Springer Nature - all rights reserved 2020

\section{Millenaar · T. Fehlmann · C. Ukena · S. Scholz · A. Keller ·F. Mahfoud · M. Böhm Wissenschaftslandschaft Deutschland}

\section{Zusammenfassung}

In der vorliegenden Analyse wurde die Entwicklung der kardiovaskulären Forschung in Deutschland seit der Wiedervereinigung analysiert. Hierbei wurden durch geeignete Suchtermini insgesamt 51.430 Publikationen aus deutschen Instituten zwischen 1990 und 2018 bei Web of Science identifiziert und szientometrisch analysiert. Die Analyse erfolgte für die gesamte Bundesrepublik sowie für alle Bundesländer getrennt. Bei der Auswertung wurde einerseits die Gesamtanzahl aller Publikationen berücksichtigt, andererseits wurde mit dem $\mathrm{H}$-Index die Publikationsqualität untersucht. Diese Ergebnisse wurden in Relation zu Einwohnerzahl, wissenschaftlicher (Anzahl medizinischer Fakultäten) sowie finanzieller (Bruttoinlandsprodukt, Höhe der Drittmittel) Ressourcen analysiert. In einer gesonderten Analyse wurde die Geschlechterverteilung der Erstautorenschaften aller Publikationen betrachtet, bei der sich ein Anteil an weiblichen Autoren von 19,4\% zeigte, während
43,9\% federführend durch männliche Wissenschaftler veröffentlicht wurden (bei $36,7 \%$ blieb die Geschlechterzuordnung unklar). Hierbei konnte ein Zuwachs des Frauenanteils von 19,8\% (Jahr 1990) auf 33,1 \% (Jahr 2018) im Vergleich zum Männeranteil beobachtet werden. Weiterhin konnten in themenspezifischen Analysen deutliche Unterschiede nachgewiesen werden. So zeigte sich im Themengebiet der koronaren Herzkrankheit konstant die höchste Anzahl an Publikationen, während die Zahlen der Publikationen zu Klappenvitien und Herzrhythmusstörungen in den letzten Jahren deutlich zunahmen. Insgesamt zeichneten sich die Forschungsaktivitäten in Deutschland durch eine besondere Kooperationsfreudigkeit mit anderen Ländern aus.

\section{Schlüsselwörter}

Forschungseffizienz - Deutschland . Kardiologie · Szientometrie $\cdot$ Hirsch-Index

\section{Scientific landscape in Germany}

\section{Abstract}

In this study the development of cardiovascular research in Germany after the reunification was analyzed. Appropriate search terms were used to identify a total of 51,430 articles from German research institutes between 1990 and 2018 in Web of Science, which were analyzed by scientometrics. The analysis was performed for the whole of Germany as well as for each federal state separately and included both publication quantity in terms of total number of publications and quality as expressed by the $\mathrm{H}$-index. These results were assessed in relation to the number of inhabitants, scientific resources (number of medical faculties) and financial resources (gross domestic product, size of third-party funding). In a separate analysis the proportions of male and female researchers as first authors in all articles were analyzed. This revealed that $19.4 \%$ of all articles were published by female researchers, whereas 43.9\% were published by male scientists (in $36.7 \%$ the gender assignment was unclear). There were an increased number of articles by female first authors from $19.8 \%$ in 1990 to $33.1 \%$ in 2018 compared to the male proportion. Moreover, in topicspecific analyses clear differences could be identified. Coronary artery disease remained the most prevalent topic for publications, while the number of publications on valvular heart diseases and arrhythmias has clearly increased in recent years. Overall the research activities in Germany were characterized by a strong cooperation with other countries.

\section{Keywords}

Research efficiency · Germany · Cardiology · Scientometry $\cdot$ Hirsch-Index 
Tab. 1 Übersicht über Einwohnerzahl (Stand 2018), Gesamtpublikationsleistung und modifizierten durchschnittlichen Hirsch(H)-Index aller kardiologischen Artikel bis 2018 sowie der Anzahl medizinischer Fakultäten (nur staatliche Universitäten), des Bruttoinlandsproduktes (Stand 2018) [1] sowie der finanziellen Drittmittel (Stand 2017) [2]. Gewertet wurden nur Erstautoren aus deutschen Instituten

\begin{tabular}{|c|c|c|c|c|c|c|c|}
\hline & Bundesland & $\begin{array}{l}\text { Einwohnerzahl } \\
\text { (in Tausend) }\end{array}$ & $\begin{array}{l}\text { Publika- } \\
\text { tionen }\end{array}$ & $\begin{array}{l}\text { Maximaler } \\
\text { H-Index }\end{array}$ & $\begin{array}{l}\text { Anzahl medizini- } \\
\text { scher Fakultäten }\end{array}$ & $\begin{array}{l}\text { Bruttoinlandsprodukt } \\
\text { (in Mio. } € \text { ) }\end{array}$ & $\begin{array}{l}\text { Drittmittel } \\
\text { (in Mio. } € \text { ) }\end{array}$ \\
\hline 1 & Baden-Württemberg & 11.070 & 1978 & 159 & 5 & 511.420 & 1305 \\
\hline 2 & Bayern & 13.077 & 2544 & 202 & 6 & 625.161 & 1205 \\
\hline 3 & Berlin & 3645 & 1404 & 143 & 1 & 147.057 & 633 \\
\hline 4 & Brandenburg & 2512 & 149 & 52 & 0 & 73.722 & 125 \\
\hline 5 & Bremen & 683 & 40 & 43 & 0 & 34.294 & 146 \\
\hline 6 & Hamburg & 1841 & 712 & 107 & 1 & 120.332 & 286 \\
\hline 7 & Hessen & 6266 & 1055 & 135 & 3 & 292.016 & 522 \\
\hline 8 & Mecklenburg-Vorpommern & 1610 & 320 & 73 & 2 & 44.924 & 106 \\
\hline 9 & Niedersachsen & 7982 & 1046 & 130 & 2 & 296.016 & 635 \\
\hline 10 & Nordrhein-Westfalen & 17.933 & 2667 & 140 & 7 & 705.066 & 1586 \\
\hline 11 & Rheinland-Pfalz & 4085 & 630 & 110 & 1 & 149.148 & 248 \\
\hline 12 & Saarland & 991 & 289 & 103 & 1 & 35.961 & 80 \\
\hline 13 & Sachsen & 4078 & 927 & 138 & 2 & 126.364 & 536 \\
\hline 14 & Sachsen-Anhalt & 2208 & 225 & 73 & 2 & 63.504 & 133 \\
\hline 15 & Schleswig-Holstein & 2897 & 455 & 107 & 2 & 97.074 & 165 \\
\hline 16 & Thüringen & 2143 & 34 & 20 & 1 & 63.804 & 175 \\
\hline
\end{tabular}

tionen mit der Anzahl der Einwohner und medizinischer Fakultäten korreliert. Die größte Anzahl an Publikationen ist Nordrhein-Westfalen zuzurechnen, dem bevölkerungsreichsten Bundesland mit einer hohen Anzahl an medizinischen Fakultäten sowie dem größten Bruttoinlandsprodukt (BIP) und der höchsten Summe an erhaltenen Drittmittel. Weniger bevölkerungsreiche Bundesländer weisen dementsprechend eine geringere Quantität der Publikationen auf. Die - Abb. 2b visualisiert den maximalen H-Index jedes Bundeslandes und ist somit ein Parameter für die Forschungsgüte des entsprechenden Bundeslandes.

I) Die Anzahl der Publikationen korreliert mit der Anzahl an Einwohnern und medizinischen Fakultäten

Bei der Einordnung der wissenschaftlichen Leistung sollte ebenso die Einwohnerzahl der jeweiligen Bundesländer mit einbezogen werden, um entsprechende Größenunterschiede zu berücksichtigen. Eine noch genauere Einschätzung der Menge sowie der Qualität der For- schungsleistung kann unter Berücksichtigung der Anzahl der medizinischen Fakultäten pro Bundesland erfolgen. Weiterhin erfolgten Analysen der Publikationseffizienz in Bezug auf finanzielle Ressourcen, sowohl des individuellen Bruttoinlandsproduktes als auch der Höhe der Drittmittelbeträge der forschenden Einrichtungen, wie in • Tab. 1 aufgeführt.

Durch die Berücksichtigung der Einwohnerstärke sowie der wissenschaftlichen und finanziellen Ressourcen konnten somit zusätzlich zur Größe des Bundeslandes weitere relevante Faktoren berücksichtigt werden. Zur besseren Veranschaulichung erfolgte daher ein Ranking aller Bundesländer unter Berücksichtigung oben genannter Charakteristika in Bezug auf Anzahl der Publikationen sowie des H-Indexes (- Tab. 2). Hierdurch wurden insbesondere Bundesländer hervorgehoben, die trotz geringer Einwohnerzahl oder niedriger Zahl medizinischer Fakultäten qualitativ hochwertige Forschungsleistung betreiben. Die - Abb. 3 visualisiert die Publikationszahl sowie den maximalen H-Index in Relation zur Anzahl medizinischer Fakultäten. Hierbei sind insbesondere die Bundesländer im oberen Bereich zu finden, die nur eine
Fakultät vorweisen können, wie Berlin, Hamburg, Rheinland-Pfalz, Thüringen und das Saarland. Da es in Brandenburg und Bremen keine medizinischen Fakultäten gibt, wurden diese beiden Bundesländer in der entsprechenden Analyse nicht berücksichtigt.

Eine weitere Einschätzung der Forschungseffizienz kann mittels finanzieller Ressourcen erfolgen. Hierzu wurden erneut die Parameter Publikationsanzahl und H-Index in Relation gesetzt $\mathrm{zu}$ einerseits Bruttoinlandsprodukt (BIP) (- Abb. 4, Stand 2018) und andererseits Höhe an zur Verfügung stehenden Drittmitteln der einzelnen Institute (• Abb. 5, Stand 2017). Unter Drittmittel wurden sämtliche finanzielle Drittmitteleinnahmen der Hochschulen laut Bericht des Statistischen Bundesamtes von 2017 berücksichtigt sowohl Zuführungsbeträge von Bund und Ländern als auch von der Europäischen Union, der Deutschen Forschungsgemeinschaft sowie Drittmittel aus Stiftungen oder der gewerblichen Wirtschaft. Diese Analyse dient der Information, wie effizient einzelne Institute mit den ihnen zur Verfügung stehenden Mitteln arbeiten können. 

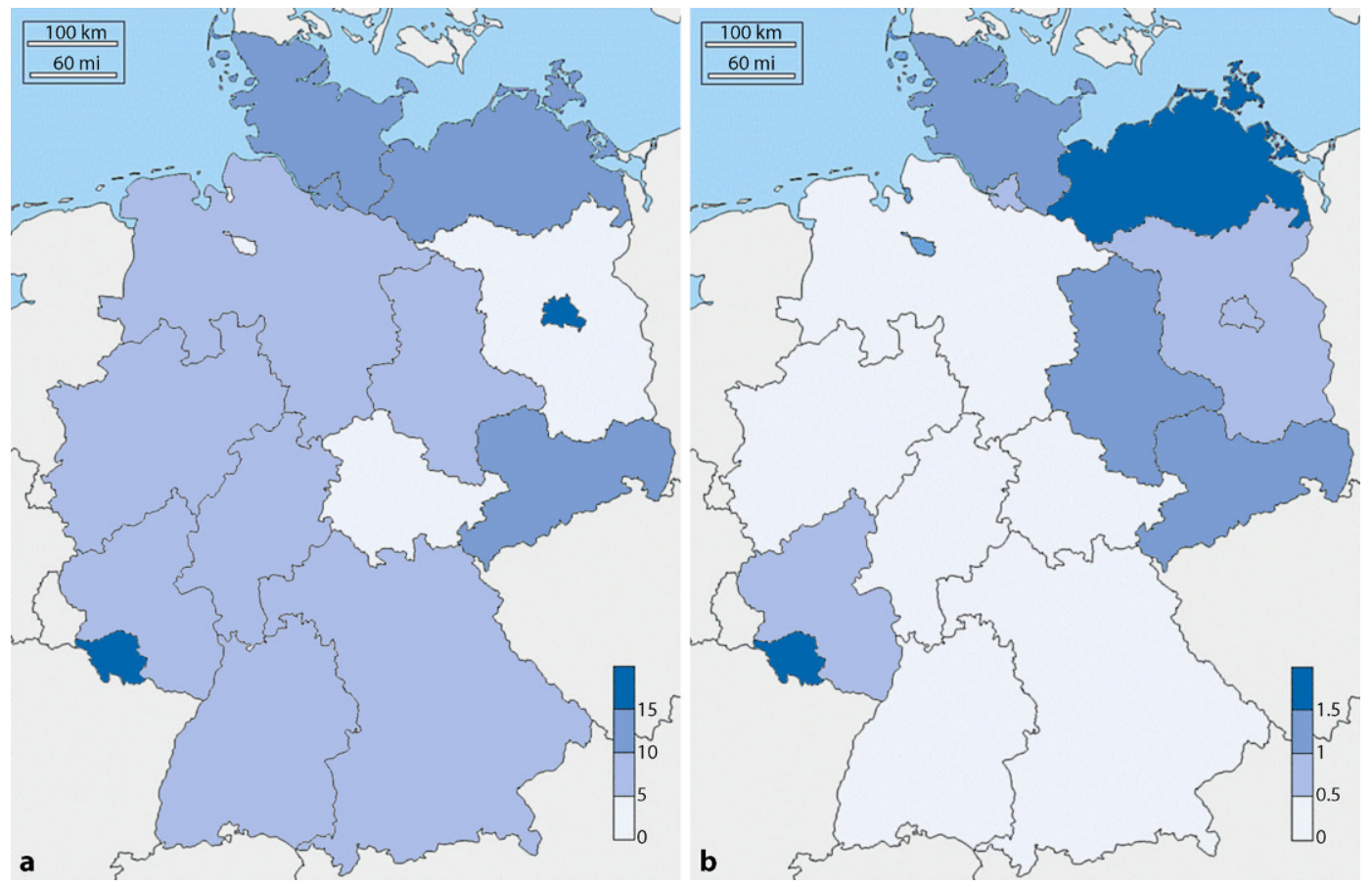

Abb. $4<$ Darstellung der Gesamtanzahl an kardiologischen Publikationen (a) sowie des modifizierten Hirsch(H)-Indexes (b) in $\mathrm{Re}$ lation zum Bruttoinlandsprodukt des jeweiligen Bundeslandes bis 2018. Farbkodierung entsprechend der jeweiligen Legende
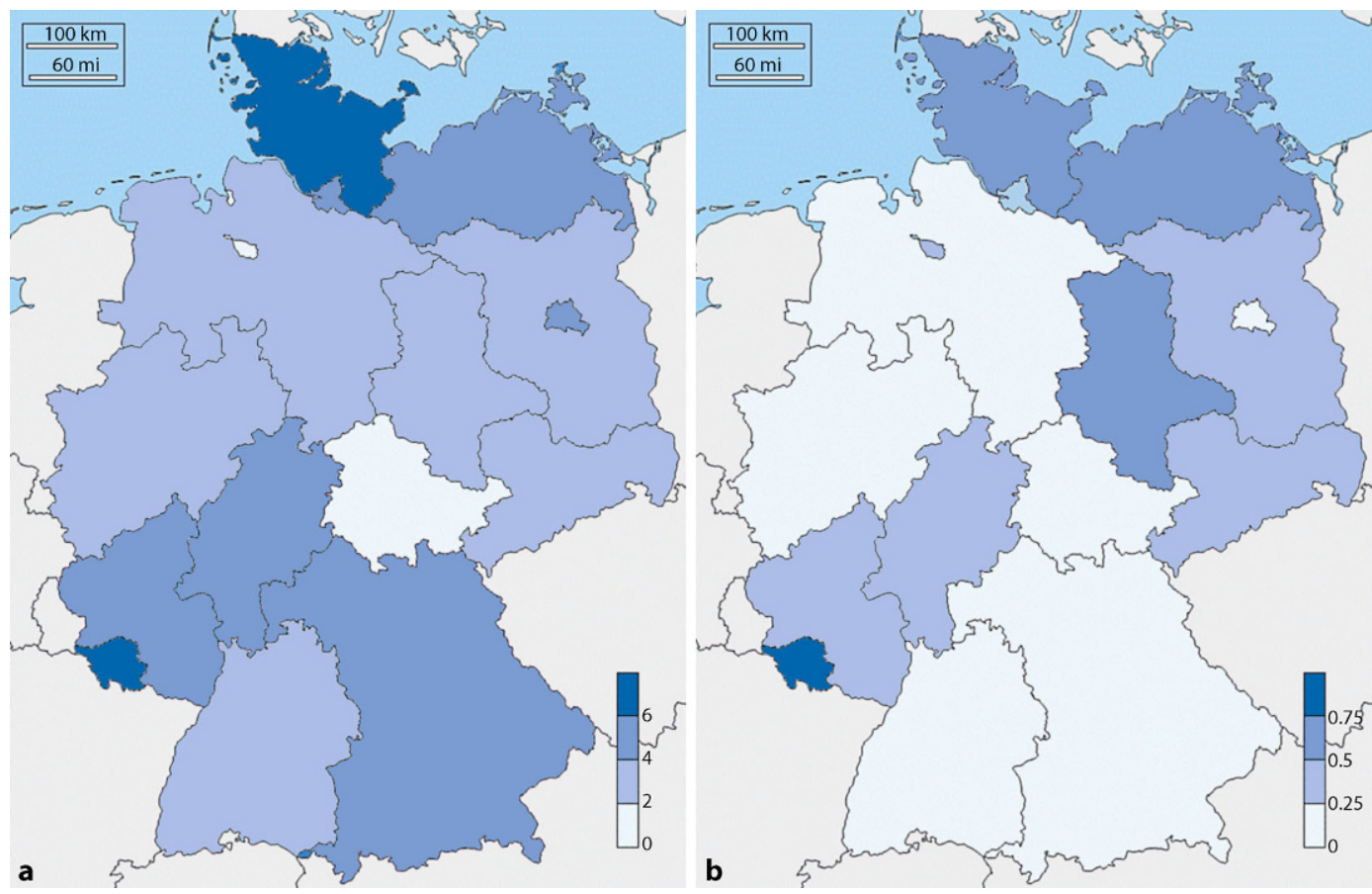

Abb. $5<$ Darstellung der Gesamtanzahl an kardiologischen Publikationen (a) sowie des modifizierten Hirsch(H)-Indexes (b) in Relation zur Hochschulfinanzierung gemessen an der Höhe der Drittmittel des jeweiligen Bundeslandes bis 2018. Farbkodierung entsprechend der jeweiligen Legende

\section{Geschlechterverteilung in der kardiologischen Forschung}

Eine Analyse der Geschlechterverteilung der Erstautorenschaft in der kardiologischen Forschung ergab einen deutlichen Unterschied zwischen Frauen und Männern. Der Gesamtanteil der Frauen betrug über den gesamten Zeitraum 19,4\%, der der Männer 43,9\% (• Abb. 6). Da die
Analyse automatisiert durch geschlechterspezifische Zuordnung der Vornamen erfolgte, blieb die Zuordnung bei 36,7\% unklar. Dies war beispielsweise der Fall, wenn nur die Initialen der Vornamen angegeben wurden und somit keine klare Zuordnung ermöglicht wurde. Insgesamt gab es einen deutlichen Anstieg bei Frauen wie auch Männern, wohingegen die Zunahme der wissenschaftlichen Aktivi- tät deutlich stärker ausgeprägt war. So betrug der Anteil der weiblichen Erstautorenschaften im Jahr 1990 19,8 \% des Männeranteils und im Jahre 2000 17,6\%. Bis 2010 war der Anteil bereits auf 26,1 \% angestiegen und lag 2018 bereits bei 33,9\%. Auch der absolute Zuwachs an Wissenschaftsleistung war bei den Wissenschaftlerinnen im Vergleich deutlich höher: Während es im Jahr 1990 noch 61 Arti- 


\section{Schwerpunkt}
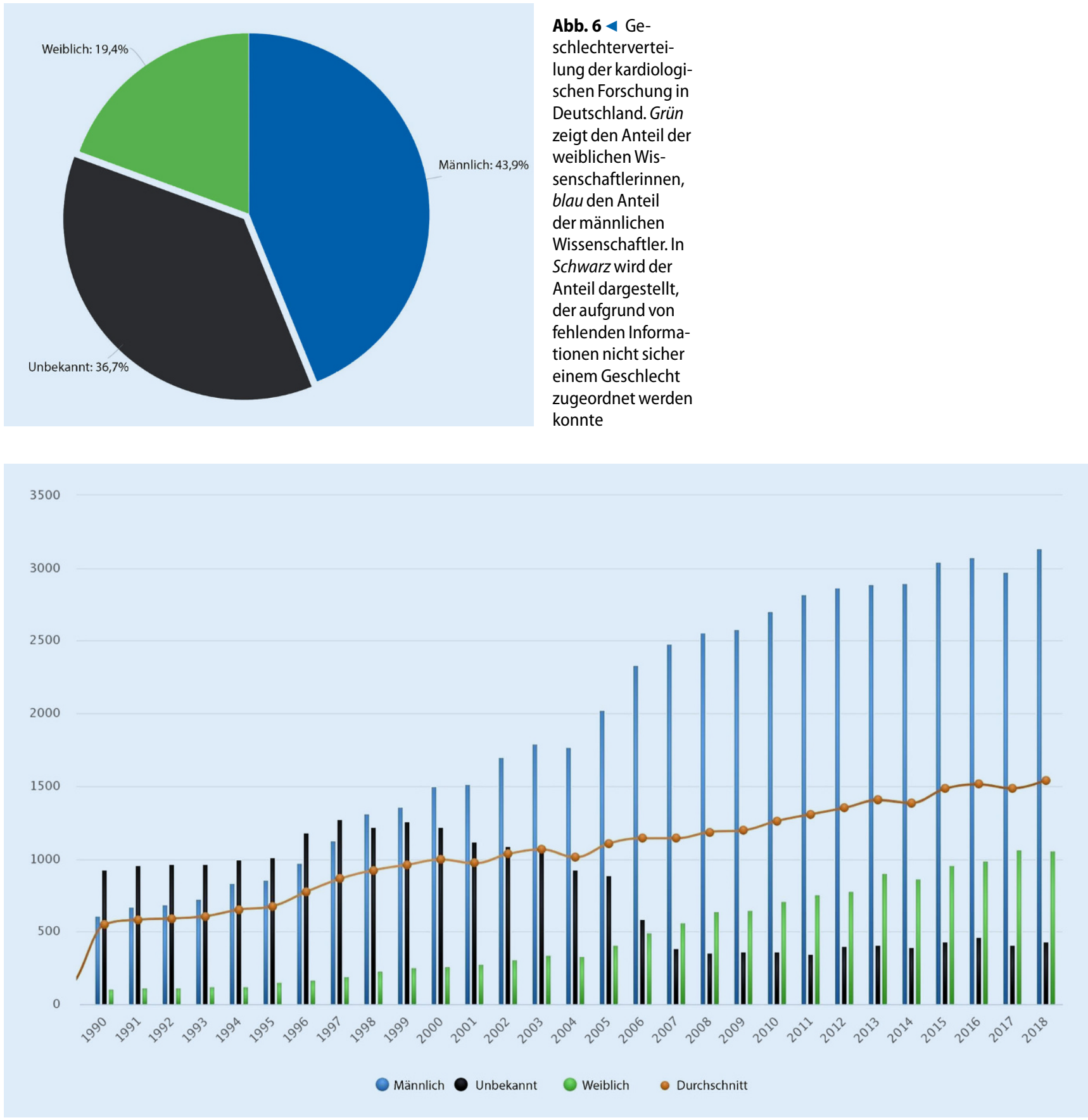

Abb. 7 A Geschlechterverteilung der kardiologischen Forschung in Deutschland getrennt nach einzelnen Jahren seit 1990. Aufgezeigt ist die Gesamtanzahl kardiologischer Publikationen in Deutschland sowohl für die beiden Geschlechter weiblich (grün), männlich (blau) als auch für die nicht klassifizierbaren (schwarz). In Orange ist der Gesamtdurchschnitt der Wissenschaftsleistung unabhängig vom Geschlecht aufgeführt

kel von Erstautorinnen waren, betrug die Anzahl im Jahr 2018 bereits 991 Artikel, entsprechend einer Zunahme um etwa das 16-Fache. Bei den männlichen Erstautoren kam es zu einem Anstieg von 308 Artikeln (Jahr 1990) auf 2925 Artikel (Jahr 2018) und somit zu einem Anstieg um das knapp 10-Fache (• Abb. 7).

\section{Verteilung in Deutschland}

Da die kardiovaskuläre Forschung aus vielen verschiedenen Teilbereichen besteht, erfolgte eine separate Auswertung aller großen Teilbereiche wie Herzrhythmusstörungen, Herzinsuffizienz, arterielle Hypertonie, infektiöse Herzerkran- kungen, koronare Herzkrankheit (KHK), periphere arterielle Verschlusskrankheit (pAVK), pulmonale Hypertonie und Klappenvitien. Die - Abb. 8 bildet den Verlauf der Forschungsleistung der einzelnen Themenbereiche für Deutschland seit 1990 ab. Hierbei zeigte sich ein deutlicher Anstieg aller Themenbereiche mit 
Tab. 2 Darstellung aller Bundesländer sortiert nach Publikationen bzw. maximalem Hirsch(H)-Index pro Einwohnerzahl, medizinischer Fakultät, Bruttoinlandsprodukt [1] sowie finanziellen Drittmitteln [2]

\begin{tabular}{|c|c|c|c|c|c|c|c|c|}
\hline \multirow[t]{2}{*}{ Rang } & $\begin{array}{l}\text { Publika- } \\
\text { tionen }\end{array}$ & H-Index & Publikationen & H-Index & $\begin{array}{l}\text { Publika- } \\
\text { tionen }\end{array}$ & H-Index & $\begin{array}{l}\text { Publika- } \\
\text { tionen }\end{array}$ & H-Index \\
\hline & \multicolumn{2}{|c|}{... pro 1 Mio. Einwohner } & \multicolumn{2}{|c|}{$\begin{array}{l}\text {... pro Medizinischer Fakultät } \\
\text { (nur deutsche, öffentliche Uni) }\end{array}$} & \multicolumn{2}{|c|}{$\begin{array}{l}\text {... pro Bruttoinlands- } \\
\text { produkt }\end{array}$} & \multicolumn{2}{|c|}{... pro Mio. $€$ Drittmitte } \\
\hline 1 & $\begin{array}{l}\mathrm{BE} \\
(816)\end{array}$ & $\begin{array}{l}\text { HB } \\
(768)\end{array}$ & $\begin{array}{l}\text { BE } \\
(2973)\end{array}$ & $\begin{array}{l}\text { BE } \\
(143)\end{array}$ & $\begin{array}{l}\mathrm{BE} \\
(20,2)\end{array}$ & $\begin{array}{l}\text { SL } \\
(2,86)\end{array}$ & $\begin{array}{l}\text { SL } \\
(6,9)\end{array}$ & $\begin{array}{l}\text { SL } \\
(1,28)\end{array}$ \\
\hline 2 & $\begin{array}{l}\mathrm{HH} \\
(696)\end{array}$ & $\begin{array}{l}\text { TH } \\
(488)\end{array}$ & $\begin{array}{l}\text { RP } \\
(1368)\end{array}$ & $\begin{array}{l}\text { RP } \\
(110)\end{array}$ & $\begin{array}{l}\text { SL } \\
(15,5)\end{array}$ & $\begin{array}{l}\text { MV } \\
(1,63)\end{array}$ & $\begin{array}{l}\text { SH } \\
(6,0)\end{array}$ & $\begin{array}{l}\text { MV } \\
(0,69)\end{array}$ \\
\hline 3 & $\begin{array}{l}\text { SL } \\
(563)\end{array}$ & $\begin{array}{l}\text { SL } \\
(185)\end{array}$ & $\begin{array}{l}\mathrm{HH} \\
(1282)\end{array}$ & $\begin{array}{l}\mathrm{HH} \\
(107)\end{array}$ & $\begin{array}{l}\text { SN } \\
(12,5)\end{array}$ & $\begin{array}{l}\mathrm{HB} \\
(1,25)\end{array}$ & $\begin{array}{l}\mathrm{RP} \\
(5,5)\end{array}$ & $\begin{array}{l}\text { SH } \\
(0,65)\end{array}$ \\
\hline 4 & $\begin{array}{l}\text { BY } \\
(420)\end{array}$ & $\begin{array}{l}\text { BB } \\
(184)\end{array}$ & $\begin{array}{l}\mathrm{NI} \\
(980)\end{array}$ & $\begin{array}{l}\text { SL } \\
(103)\end{array}$ & $\begin{array}{l}\text { MV } \\
(11,4)\end{array}$ & $\begin{array}{l}\text { ST } \\
(1,15)\end{array}$ & $\begin{array}{l}\text { MV } \\
(4,8)\end{array}$ & $\begin{array}{l}\text { ST } \\
(0,55)\end{array}$ \\
\hline 5 & $\begin{array}{l}\text { SN } \\
(387)\end{array}$ & $\begin{array}{l}\text { ST } \\
(151)\end{array}$ & $\begin{array}{l}\text { BY } \\
(916)\end{array}$ & $\begin{array}{l}\text { SN } \\
(69)\end{array}$ & $\begin{array}{l}\mathrm{HH} \\
(10,7)\end{array}$ & $\begin{array}{l}\text { SH } \\
(1,10)\end{array}$ & $\begin{array}{l}\mathrm{BE} \\
(4,7)\end{array}$ & $\begin{array}{l}\mathrm{RP} \\
(0,44)\end{array}$ \\
\hline 6 & $\begin{array}{l}\text { HE } \\
(385)\end{array}$ & $\begin{array}{l}\text { MV } \\
\text { (143) }\end{array}$ & $\begin{array}{l}\text { NW } \\
(873)\end{array}$ & $\begin{array}{l}\mathrm{NI} \\
(65)\end{array}$ & $\begin{array}{l}\text { SH } \\
(10,1)\end{array}$ & $\begin{array}{l}\text { SN } \\
(1,09)\end{array}$ & $\begin{array}{l}\mathrm{HE} \\
(4,6)\end{array}$ & $\begin{array}{l}\text { BB } \\
(0,42)\end{array}$ \\
\hline 7 & $\begin{array}{l}\text { BW } \\
(382)\end{array}$ & $\begin{array}{l}\text { SH } \\
(109)\end{array}$ & $\begin{array}{l}\text { BW } \\
(846)\end{array}$ & $\begin{array}{l}\text { SH } \\
(54)\end{array}$ & $\begin{array}{l}\text { RP } \\
(9,2)\end{array}$ & $\begin{array}{l}\mathrm{BE} \\
(0,97)\end{array}$ & $\begin{array}{l}\text { BY } \\
(4,6)\end{array}$ & $\begin{array}{l}\mathrm{HH} \\
(0,37)\end{array}$ \\
\hline 8 & $\begin{array}{l}\text { NW } \\
(341)\end{array}$ & $\begin{array}{l}\text { SN } \\
(87)\end{array}$ & $\begin{array}{l}\mathrm{HE} \\
(805)\end{array}$ & $\begin{array}{l}\text { HE } \\
(45)\end{array}$ & $\begin{array}{l}\text { BY } \\
(8,8)\end{array}$ & $\begin{array}{l}\mathrm{HH} \\
(0,89)\end{array}$ & $\begin{array}{l}\mathrm{HH} \\
(4,5)\end{array}$ & $\begin{array}{l}\text { HB } \\
(0,29)\end{array}$ \\
\hline 9 & $\begin{array}{l}\text { SH } \\
\text { (339) }\end{array}$ & $\begin{array}{l}\mathrm{HH} \\
(83)\end{array}$ & $\begin{array}{l}\text { SN } \\
\text { (789) }\end{array}$ & $\begin{array}{l}\text { MV } \\
\text { (37) }\end{array}$ & $\begin{array}{l}\text { NW } \\
(8,7)\end{array}$ & $\begin{array}{l}\text { RP } \\
(0,74)\end{array}$ & $\begin{array}{l}\text { NW } \\
(3,9)\end{array}$ & $\begin{array}{l}\mathrm{HE} \\
(0,26)\end{array}$ \\
\hline 10 & $\begin{array}{l}\text { RP } \\
\text { (335) }\end{array}$ & $\begin{array}{l}\text { RP } \\
(80)\end{array}$ & $\begin{array}{l}\text { SL } \\
\text { (558) }\end{array}$ & $\begin{array}{l}\text { ST } \\
\text { (37) }\end{array}$ & $\begin{array}{l}\text { BW } \\
(8,3)\end{array}$ & $\begin{array}{l}\text { BB } \\
(0,71)\end{array}$ & $\begin{array}{l}\text { ST } \\
(3,6)\end{array}$ & $\begin{array}{l}\text { SN } \\
(0,26)\end{array}$ \\
\hline 11 & $\begin{array}{l}\text { MV } \\
(318)\end{array}$ & $\begin{array}{l}\mathrm{NI} \\
(66)\end{array}$ & $\begin{array}{l}\text { SH } \\
(492)\end{array}$ & $\begin{array}{l}\text { BY } \\
\text { (34) }\end{array}$ & $\begin{array}{l}\text { HE } \\
(8,3)\end{array}$ & $\begin{array}{l}\text { HE } \\
(0,46)\end{array}$ & $\begin{array}{l}\text { BW } \\
(3,2)\end{array}$ & $\begin{array}{l}\mathrm{BE} \\
(0,23)\end{array}$ \\
\hline 12 & $\begin{array}{l}\mathrm{NI} \\
(245)\end{array}$ & $\begin{array}{l}\text { HE } \\
(56)\end{array}$ & $\begin{array}{l}\text { MV } \\
(256)\end{array}$ & $\begin{array}{l}\text { BW } \\
\text { (32) }\end{array}$ & $\begin{array}{l}\text { ST } \\
(7,6)\end{array}$ & $\begin{array}{l}\mathrm{NI} \\
(0,44)\end{array}$ & $\begin{array}{l}\mathrm{NI} \\
(3,1)\end{array}$ & $\begin{array}{l}\mathrm{NI} \\
(0,20)\end{array}$ \\
\hline 13 & $\begin{array}{l}\text { ST } \\
(218)\end{array}$ & $\begin{array}{l}\text { BE } \\
(48)\end{array}$ & $\begin{array}{l}\text { ST } \\
(241)\end{array}$ & $\begin{array}{l}\text { TH } \\
(20)\end{array}$ & $\begin{array}{l}\mathrm{NI} \\
(6,6)\end{array}$ & $\begin{array}{l}\text { BY } \\
(0,32)\end{array}$ & $\begin{array}{l}\text { SN } \\
(2,9)\end{array}$ & $\begin{array}{l}\text { BY } \\
(0,20)\end{array}$ \\
\hline 14 & $\begin{array}{l}\text { BB } \\
(112)\end{array}$ & $\begin{array}{l}\text { BW } \\
\text { (38) }\end{array}$ & $\begin{array}{l}\text { TH } \\
(41)\end{array}$ & $\begin{array}{l}\text { NW } \\
(20)\end{array}$ & $\begin{array}{l}\text { BB } \\
(3,8)\end{array}$ & $\begin{array}{l}\text { TH } \\
(0,31)\end{array}$ & $\begin{array}{l}\text { BB } \\
(2,3)\end{array}$ & $\begin{array}{l}\text { BW } \\
(0,12)\end{array}$ \\
\hline 15 & $\begin{array}{l}\mathrm{HB} \\
(82)\end{array}$ & $\begin{array}{l}\text { BY } \\
\text { (37) }\end{array}$ & $\begin{array}{l}\text { BB } \\
(-)\end{array}$ & $\begin{array}{l}\text { BB } \\
(-)\end{array}$ & $\begin{array}{l}\text { HB } \\
(1,6)\end{array}$ & $\begin{array}{l}\text { BW } \\
(0,31)\end{array}$ & $\begin{array}{l}\text { HB } \\
(0,4)\end{array}$ & $\begin{array}{l}\text { TH } \\
(0,11)\end{array}$ \\
\hline 16 & $\begin{array}{l}\text { TH } \\
(19)\end{array}$ & $\begin{array}{l}\text { NW } \\
(23)\end{array}$ & $\begin{array}{l}\text { HB } \\
(-)\end{array}$ & $\begin{array}{l}\text { HB } \\
(-)\end{array}$ & $\begin{array}{l}\text { TH } \\
(0,6)\end{array}$ & $\begin{array}{l}\text { NW } \\
(0,20)\end{array}$ & $\begin{array}{l}\text { TH } \\
(0,2)\end{array}$ & $\begin{array}{l}\text { NW } \\
(0,09)\end{array}$ \\
\hline
\end{tabular}

$B B$ Brandenburg, BE Berlin, BW Baden-Württemberg, BY Bayer, $H E$ Hessen, HB Bremen, $H H$ Hamburg, MV Mecklenburg-Vorpommern, NI Niedersachsen, NW Nordrhein-Westfalen, RP Rheinland-Pfalz, SH Schleswig-Holstein, SN Sachsen, SL Saarland, ST Sachsen-Anhalt, TH Thüringen

deutlichem Vorsprung im Bereich der KHK und Koronarintervention. Dies spiegelt zum einen die hohe Prävalenz dieser Erkrankung wider, ist aber ebenso Ausdruck der anhaltenden wissenschaftlichen Relevanz dieses Themengebietes. Ein deutlicher Anstieg ist ebenfalls im Bereich der Arrhythmien sowie der Klappenvitien erkennbar, was sicherlich neben der ebenfalls steigenden Prävalenz auch auf die zunehmende Entwicklung interventioneller Verfahren dieser Bereiche zurückzuführen ist. Im Bereich der Arrhythmien kam es zu einer deutlichen Weiterentwicklung im Bereich der invasiven Elektrophysiologie, insbesondere der Katheterablationen, während im Bereich der Klappenvitien hier innovative Verfahren wie die interventionelle Therapie der Aortenklappenstenose sowie der Mitralklappeninsuffizienz Berücksichtigung finden.

\section{Meilensteine in der kardiologischen Forschung}

Im Laufe der letzten Jahre kam es zu einigen wichtigen Ereignissen in der Kardiologie, die die klinische Arbeit wesentlich verändert haben. Diese Änderungen und Neuentwicklungen spiegeln sich zugleich in der Forschungsaktivität und
Forschungsentwicklung in Deutschland wider. Die - Abb. 9 gibt einen Überblick über einige dieser Meilensteine in Bezug auf die Forschungsleistung in Deutschland. Ein kausaler Zusammenhang ist hierbei jedoch nur eingeschränkt möglich, da die Forschungsleistungen nicht linear mit den klinischen Innovationen korrelieren und eher zeitversetzt eintreten. Andererseits ist in dieser Analyse ausschließlich die deutsche Forschungsleistung aufgezeigt. Die einzelnen kardiologischen Entwicklungen dienen somit der Orientierung und der Einordnung des zeitlichen Zusammenhangs der Forschungsleistungen. 


\section{Schwerpunkt}

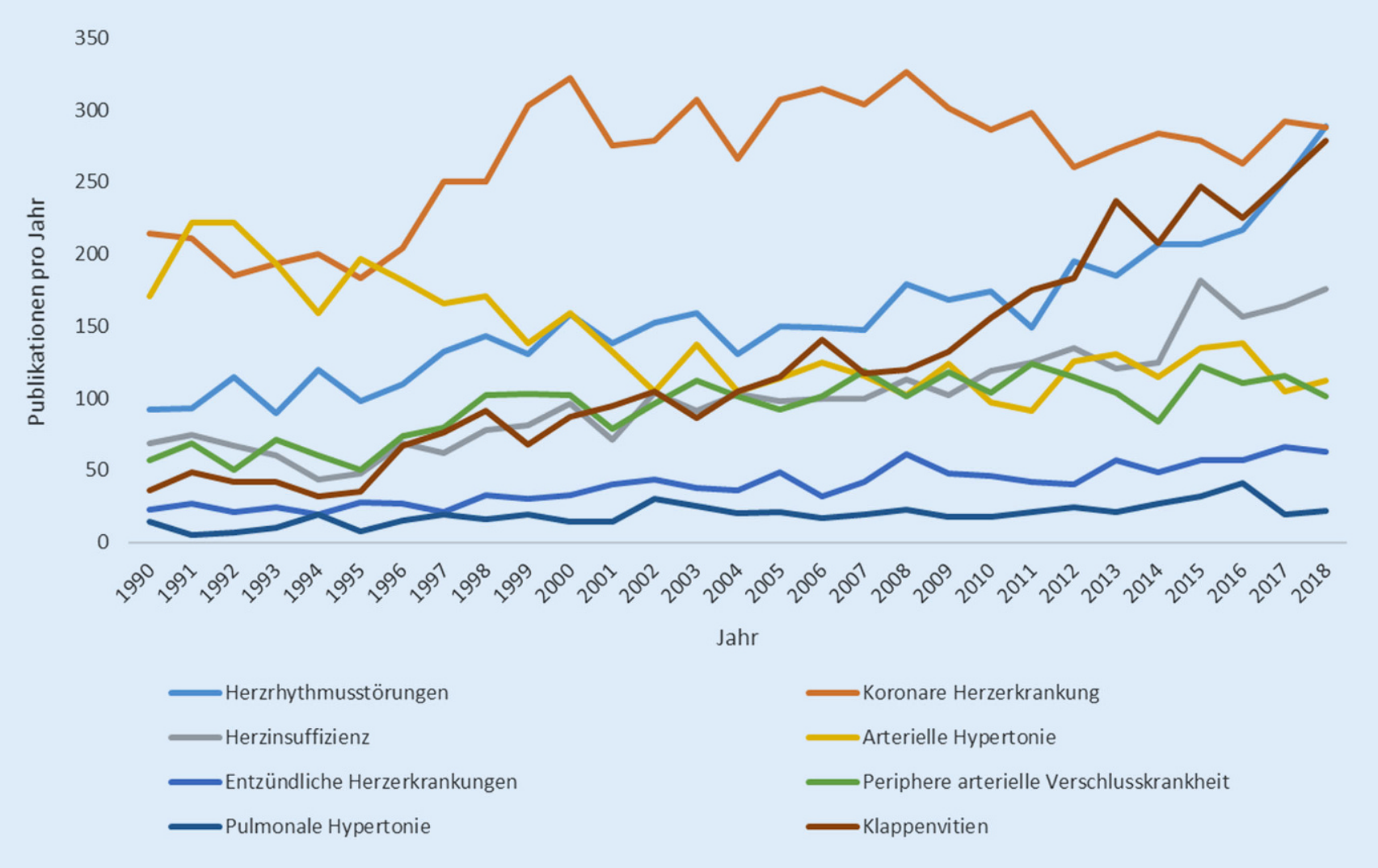

Abb. 8 A Kardiologische Forschung in Deutschland seit 1990, aufgeteilt nach verschiedenen Themenbereichen. Aufgezeigt ist die Anzahl der Publikationen pro Jahr für den jeweiligen Bereich

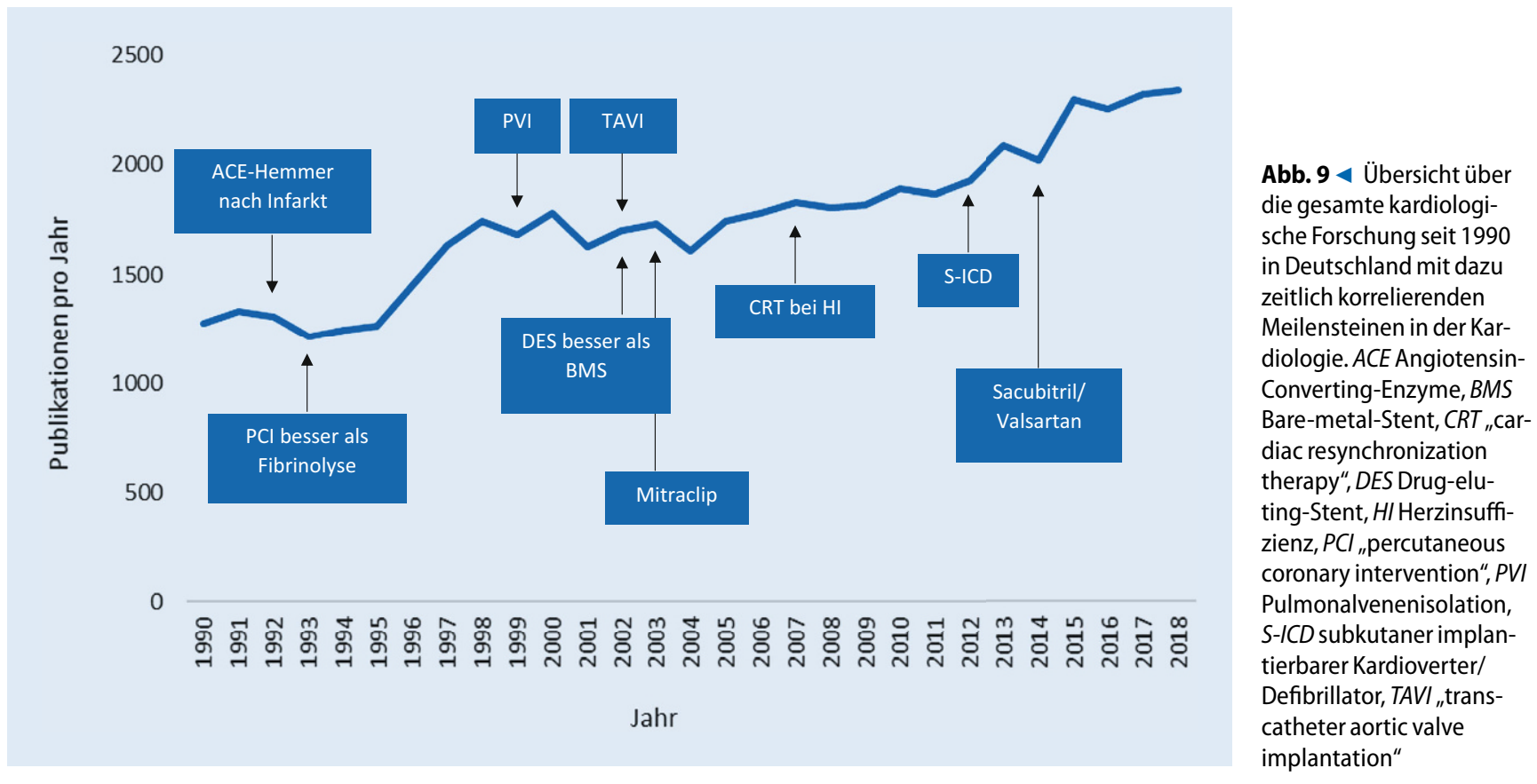




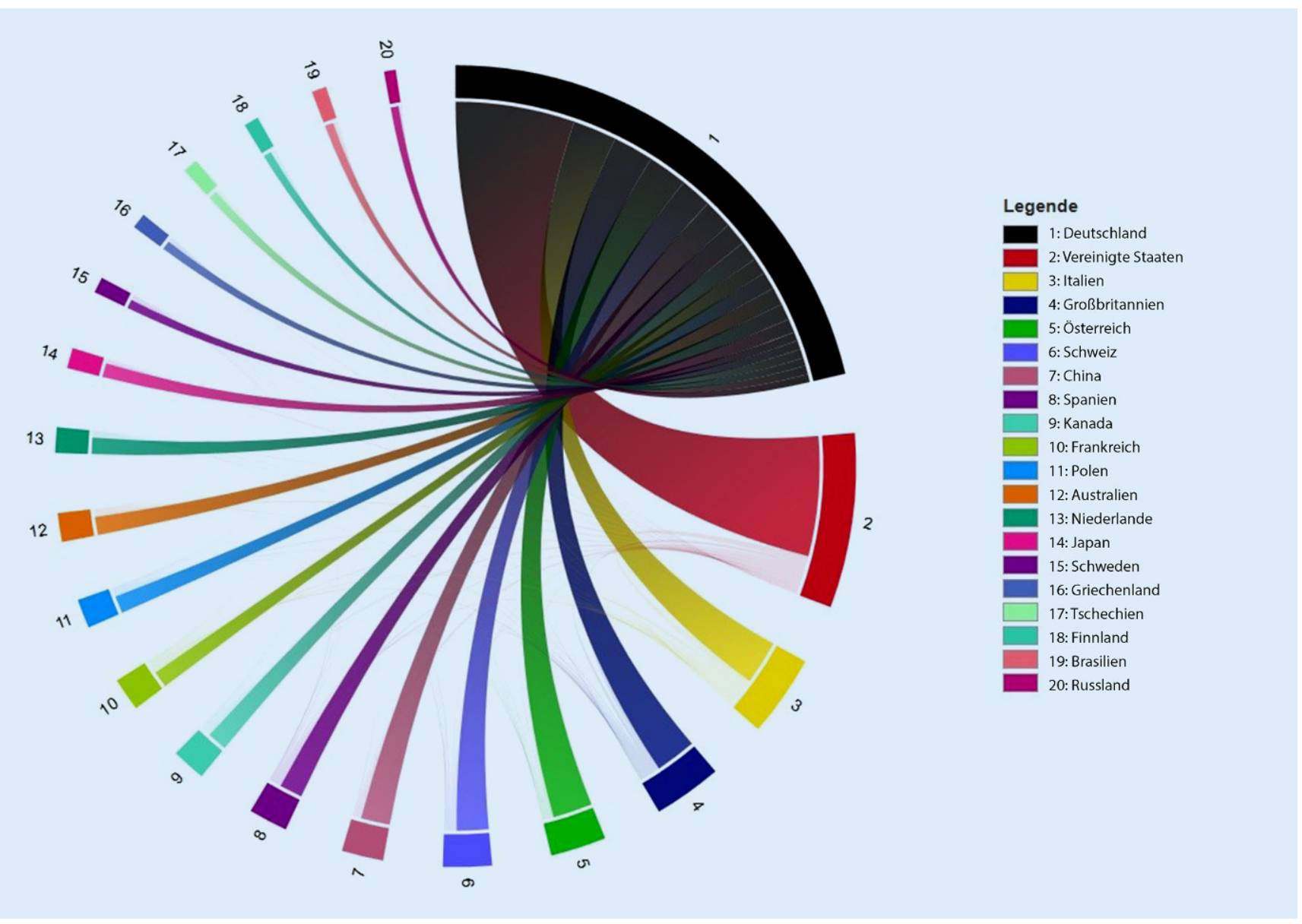

Abb. 10 ॥ Visualisierung der Kooperationen mit anderen Ländern

\section{Kooperationen}

Viele Publikationen und wissenschaftliche Projekte bestehen aus internationalen Kooperationen. Aus der Analyse der Institutszugehörigkeiten der beteiligten Autoren lässt sich das Ausmaß internationaler Kooperationen bei wissenschaftlichen Projekten ableiten. Die • Abb. 10 stellt diese Kooperationen bildlich dar. Die Breite der Verbindungslinien zwischen den Ländern ist hierbei proportional zum Ausmaß der Zusammenarbeit. Hierbei erkennt man, dass Deutschland die meisten Kooperationen mit den USA sowie Italien und Großbritannien unterhält. Kooperationen ergeben sich häufig aufgrund geografischer Nähe: So liegen beispielsweise von den 19 häufigsten Kooperationspartnern 15 Länder in Europa.

\section{Limitationen}

Bei szientometrischen Analysen sind einige Limitationen $\mathrm{zu}$ berücksichtigen. Allen voran wurden nur Artikel analysiert, die in der Online-Plattform WoS aufgeführt wurden. Trotz sorgfältiger Überprüfungen auch mit anderen medizinischen Datenbanken könnten hierdurch möglicherweise Veröffentlichungen nicht berücksichtigt worden sein. Eine weitere Einschränkung stellt der Suchterminus dar; hierbei wurde eine möglichst detaillierte und vollständige Abbildung aller kardiologischen Publikationen ausgewählt mit der größtmöglichen Signifikanz und Spezifität. Da jedoch ausschließlich über eine Titelsuche ausgewählt wurde, kann dies möglicherweise auch zu fehlenden Veröffentlichungen geführt haben. Weiterhin wurde bei der Beurteilung des Autors jeweils der Korrespondenzautor mit dessen Institutszugehörigkeit gewählt.
Hierdurch wurden große multizentrische Studien auf das Institut des Korrespondenzautors reduziert. Der H-Index steigt mit der Anzahl an Publikationen pro Land bzw. Person sowie mit der Anzahl an Zitationen. Dadurch tragen jüngere Publikationen aufgrund der noch geringen Anzahl an Zitationen noch weniger zum $\mathrm{H}$-Index bei, was wiederum bei der Bewertung des H-Indexes berücksichtigt werden muss. Da die Geschlechteranalysen mittels einer automatisierten Zuordnung des Vornamens erfolgen, können folglich ausschließlich Autorinnen und Autoren in das jeweilige Geschlecht unterteilt werden, wenn der gesamte Vorname angegeben ist und dieser auch zweifelsfrei einem Geschlecht zugeordnet werden kann.

\section{Schlussfolgerung}

Die vorliegende Analyse beschreibt erstmalig die Wissenschaftslandschaft in 
Deutschland im Bereich der kardiologischen Forschung. Insgesamt ist ein deutlicher Zuwachs von Forschung im Bereich der Kardiologie zu verzeichnen. Die Forschungseffizienz einzelner Bundesländer oder Institute kann auf verschiedene Weise ausgedrückt werden: zum einen direkt über die Quantität, der absoluten Menge an Publikationen im Bereich der Kardiologie. Zur Beurteilung der Qualität sollten jedoch weitere Parameter hinzugezogen werden, wie beispielsweise der H-Index sowie institutionelle und finanzielle Ressourcen. Durch eine differenzierte Betrachtung und Analyse der Publikationszahlen vor diesem Hintergrund kann eine Aussage über die Forschungseffizienz im Bereich der Kardiologie in Deutschland getroffen werden. Dass die Forschungsleistung jedoch nicht isoliert für einzelne Institute betrachtet werden sollte, zeigt die ausgeprägte Anzahl an wissenschaftlichen Kooperationen im internationalen Bereich, wodurch die Bedeutung von Teamwork in der Forschung erneut unterstrichen wird.

\section{Korrespondenzadresse}

\section{Dr. Dominic Millenaar}

Klinik für Innere Medizin III, Kardiologie, Angiologie und Internistische Intensivmedizin, Universitätsklinikum des Saarlandes und Medizinische Fakultät, Universität des Saarlandes

Kirrberger Str., Geb. 41.1, 66421 Homburg/Saar, Deutschland

Dominic.Millenaar@uks.eu

Danksagung. Wir danken Alexander Flohr und Markus Dillmann für die Hilfe bei der Programmierung der Software SciPE.

\section{Einhaltung ethischer Richtlinien}

Interessenkonflikt. D. Millenaar, T. FehImann, C. Ukena, S. Scholz, A. Keller, F. Mahfoud und M. Böhm geben an, dass kein Interessenkonflikt besteht.

Für diesen Beitrag wurden von den Autoren keine Studien an Menschen oder Tieren durchgeführt. Für die aufgeführten Studien gelten die jeweils dort angegebenen ethischen Richtlinien.

\section{Literatur}

1. Statistisches Bundesamt (2018) Bruttoinlandsproduktje Einwohnernach Bundesländern
2. Statistisches Bundesamt (2017) Finanzen der Hochschulen. Fachserie 11, Reihe 4.5.

3. Hirsch JE (2005) An index to quantify an individual's scientific research output. Proc Natl Acad Sci U S A 102:16569-16572

4. Scholz SS, Dillmann M, Flohr A, Backes C, Fehlmann T,MillenaarD, UkenaC, Bohm M, Keller A, Mahfoud F (2019) Contemporary scientometric analyses using a novel web application: the science performance evaluation (SciPE) approach. Clin Res Cardiol. https://doi.org/10.1007/s00392-01901568-x
Möchten Sie einen Beitrag für Der Kardiologe einreichen?

Wir freuen uns, dass Sie unsere Zeitschrift mitgestalten möchten.

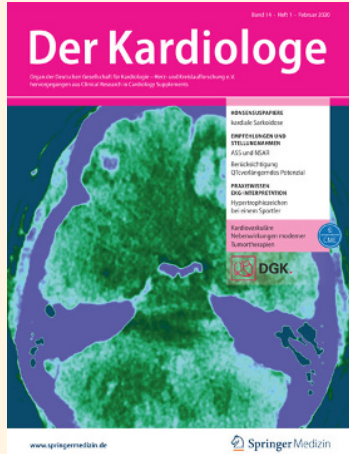

Für folgende Rubriken können Manuskripte eingereicht werden:

- Übersichten

- Originalien

- Wie lautet Ihre Diagnose?

- Praxiswissen EKG-Interpretation

Um Ihnen bei der Manuskripterstellung behilflich zu sein, haben wir für unsere Autoren ausführliche Autorenleitfäden und Musterbeiträge für die verschiedenen Rubriken zusammengestellt.

Diese und weitere Hinweise zur Manuskripterstellung finden Sie online unter dem Menüpunkt „Hinweise für Autoren" unter www.DerKardiologe.de.

Bitte reichen Sie Ihren fertigen Beitrag in elektronischer Form bei den zuständigen Schriftleitern ein

Sollten Sie noch Fragen zur Manuskriptgestaltung haben, wenden Sie sich bitte an die Redaktion:

Felix Erwin

felix.erwin@springer.com

Wir freuen uns auf Ihre Beiträge!

Ihre Redaktion von Der Kardiologe 
Hier steht eine Anzeige.

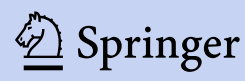

